\title{
EL GREEN NEW DEAL Y LAS FINANZAS SOSTENIBLES EN EUROPA
}

\section{GREEN NEW DEAL AND SUSTAINABLE FINANCE IN EUROPE}

\author{
Ángel Berges \\ aberges@afi.es \\ Emilio Ontiveros \\ ontiveros@afi.es \\ Catedráticos de la Universidad Autónoma de Madrid \\ y socios de Afi
}

Fecha recepción artículo: 9/03/2021 • Fecha aprobación artículo: 23/06/2021

\section{RESUMEN}

El artículo pretende aportar una visión general de la adaptación de la actividad financiera a la necesidad de un modelo de crecimiento más sostenible. Aunque la sostenibilidad incorpora también aspectos sociales y de gobernanza, el artículo se centra fundamentalmente en los relacionados con el cambio climático, sin duda el que con mayor urgencia ha demandado la adaptación por parte del sistema financiero. Sobre tales bases, el trabajo presta una atención diferencial a la modalidad de financiación sostenible más extendida, los denominados "bonos verdes", así como a las alteraciones en la actitud de los principales bloques políticos y económicos. Desde el muy relevante relevo en la presidencia estadounidense hasta el liderazgo claro de las instituciones europeas, primero con el Green New Deal, y más recientemente con el plan de recuperación verde en respuesta a la COVID-19.

Palabras clave: Cambio climático; Bonos Verdes; Taxonomía; Recuperación verde. 
Ángel Berges Lobera. Vicepresidente de Afi y Catedrático de Economía Financiera y Contabilidad en la Universidad Autónoma de Madrid, y socio fundador de AFI. Fue miembro del Stakeholders Group, tanto en la European Securities Markets Authority, (ESMA), como en la European Banking Authority (EBA), por un periodo total de diez años, entre 2011 y 2020. Autor de varios libros y numerosos artículos en revistas nacionales y extranjeras.

Emilio Ontiveros Baeza es Catedrático Emérito de Economía de la Empresa de la Universidad Autónoma de Madrid, de la que fue Vicerrector durante cuatro años, antes de fundar Afi en 1987, de la que es presidente. Autor de libros y artículos sobre economía y finanzas, en revistas académicas, profesionales y medios de comunicación. Miembro de los consejos de administración de varias empresas. Desde julio de 2020, es miembro del Consejo Asesor de Asuntos Económicos del Ministerio de Asuntos Económicos y Transformación Digital.

\section{ABSTRACT}

The article emphasizes the need for the financial system to be aligned with the necessary transition towards a more sustainable economic model. While sustainability has different dimensions (including social and governance), we concentrate our efforts on climate change, as it has attracted the need for urgent actions by financial markets and institutions, on ground of financial stability. We devote a special attention to the launching and development of Green Bonds, by far the most relevant exponent of private companies and financial markets adaptation to climate change concerns. To complement the role played by private agents, the article the develops on the leading role played by the European institutions, first through the launching of the Green New Deal, and more recently with the Green recovery initiatives in response to the COVID-19.

Keywords: Climate change; Taxonomy; Green Bonds; Green Recovery. 


\section{INTRODUCCIÓN, PRETENSIONES Y ALCANCE}

El objetivo de las notas que siguen es aportar una visión general de la adaptación de la actividad financiera a las evidencias cada día más explícitas del deterioro de las condiciones de habitabilidad del planeta como consecuencia del cambio climático. En su estructura se ha tratado de dar cuenta de ese deterioro, pero especialmente de reflejar la capacidad adaptativa de los principales operadores en los mercados financieros para hacer de la necesidad virtud y asumir en sus funciones de decisión restricciones medioambientales.

La verificación de que esas restricciones no han limitado el comportamiento en términos del binomio rentabilidad-riesgo es una conclusión relevante, cada día más amparada en observación empírica.

Sobre tales bases, el trabajo presta una atención diferencial a la modalidad de financiación sostenible más extendida, los denominados "bonos verdes", así como a las alteraciones en la actitud de los principales bloques políticos y económicos. Desde el muy relevante relevo en la presidencia estadounidense hasta el liderazgo claro de las instituciones europeas.

\section{LOS FUNDAMENTOS}

\subsection{LAS EVIDENCIAS DE DETERIORO DEL PLANETA}

A estas alturas no cabe albergar dudas sobre los perniciosos efectos que la acción humana está ejerciendo sobre el planeta y su sostenibilidad. Esos efectos, tanto directos como indirectos, se manifiestan en muchas partes del mundo, repercutiendo gravemente en el ecosistema, sobre la salud de las poblaciones y, desde luego, sobre la economía.

De forma más directa, y cuantificable, la frecuencia de eventos naturales extremos ha venido aumentando de forma ininterrumpida durante las cuatro últimas décadas, como ilustra el gráfico 1.

Gráfico 1: Aumento de eventos climáticos extremos en el mundo

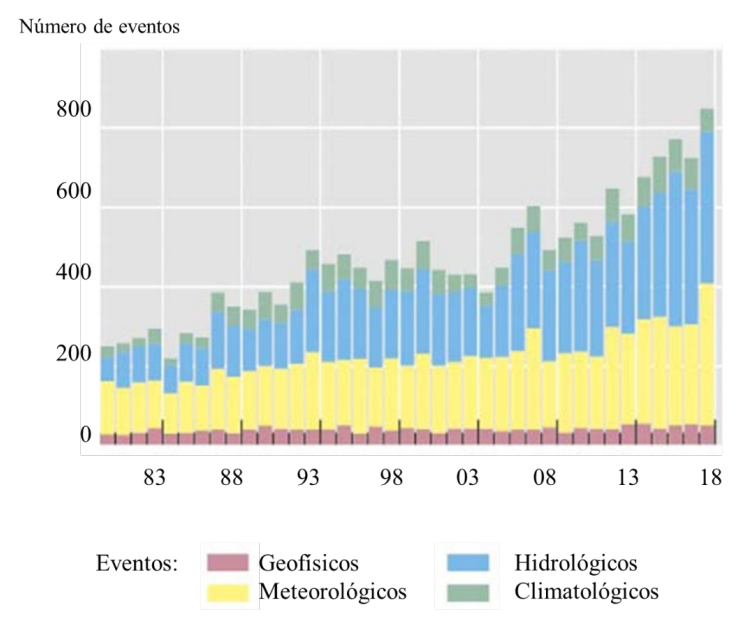

Fuente: Munich, Re (2018), obtenido de "The green swan". Central banking and financial stability in the age of climate change. BIS. Banque de France. Enero 2020 
Esos eventos extremos se han traducido en importantes pérdidas, que en los últimos años alcanzan órdenes de magnitud en el entorno de cientos de miles de millones anuales de dólares, de los que la mayor parte se encontraban sin aseguramiento alguno (gráfico 2)

\section{Gráfico 2: Pérdidas (aseguradas y no aseguradas) provocadas por eventos climáticos extremos}

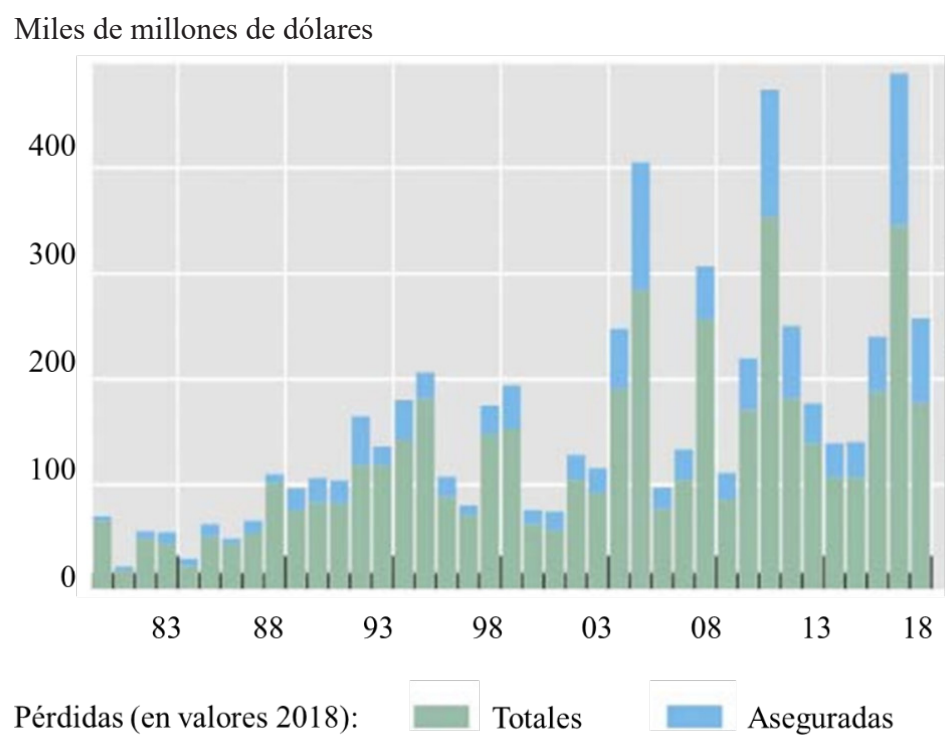

Fuente: Munich, Re (2018), obtenido de "The green swan". Central banking and financial stability in the age of climate change. BIS. Banque de France. Enero 2020

Junto a esos impactos directos causados por desastres naturales, el aumento del número de días anormalmente calurosos requiere de inversión en nuevas infraestructuras más resistentes y eficientes. Por otra parte, la literatura académica empieza a demostrar que existe una clara incidencia negativa de la creciente emergencia climática en la productividad. El calentamiento global del planeta incide de forma adversa en el rendimiento de algunos recursos naturales que genera un claro empobrecimiento de la sociedad.

En ausencia de cambios significativos en las políticas hasta ahora aplicadas, los daños físicos y económicos se incrementarán y se agudizará su irreversibilidad. El descenso de la productividad en la agricultura y en la pesca, las más frecuentes alteraciones de la actividad económica y la destrucción de capital productivo, el deterioro de la salud o directamente las pérdidas de vidas, eran destacadas por el Fondo Monetario Internacional (2020) en su último "World Economic Outlook". A ello hay que añadir la complicación en la gestión de la estabilidad macroeconómica generada por el aumento de la volatilidad de la producción y de los precios originadas por los cambios de temperatura y los desastres naturales, además de la presión sobre la sostenibilidad fiscal.

Estrechamente vinculadas a esta última son las dificultades generadas para la reducción de la pobreza y las desigualdades, no solo en las economías menos avanzadas, normalmente las más castigadas por esos desastres y las que menor margen de maniobra tienen para gestionarlos y paliar sus consecuencias sobre el bienestar. La erosión del crecimiento potencial de las economías, superior en todo caso a los cálculos que se anticiparon hace cinco años, es el denominador común a todos los escenarios que se estiman si los principales países contaminadores no cambian radical y rápidamente de comportamiento. 
En todo caso cabe resaltar que, aunque es la referida al cambio climático la acepción de sostenibilidad que mayor sensibilidad ha despertado en los últimos años, lo cierto es que el vocablo incorpora también otros significados y dimensiones adicionales a los medioambientales y estrictamente complementarios, como son los sociales y de gobernanza. En este sentido, y aunque no hay una definición única para la sostenibilidad, y en particular para las finanzas sostenibles, todas las aproximaciones al término hacen referencia a su capacidad de financiar la actividad económica "satisfaciendo las necesidades del presente sin comprometer la capacidad de las futuras generaciones para satisfacer sus propias necesidades" y favoreciendo al mismo tiempo un bienestar económico, ambiental y social.

\subsection{AGENDA DE LA ONU Y ACUERDOS DE PARÍS}

Aunque la preocupación por la sostenibilidad en esa acepción amplia viene desde muy atrás en el tiempo, lo cierto es que la misma recibió un impulso nunca antes visto de la mano de una serie de compromisos globales que marcan el camino a seguir y fijan unas metas a alcanzar. Entre ellos cabe destacar, sin duda alguna, los dos hitos que, desde 2015 , han marcado la pauta para todos los avances posteriores en materia de finanzas sostenibles: la Agenda $2030^{1}$ y el Acuerdo de París's. Si bien ambos acuerdos internacionales resultaron de negociaciones separadas, coinciden en el momento de su aprobación, y su identificación de pautas y compromisos de sostenibilidad para el futuro.

En septiembre de 2015 tuvo lugar en Naciones Unidas, y con asistencia de más de 150 jefes de Estado y de Gobierno, la histórica Cumbre del Desarrollo Sostenible, en la que se aprobó la Agenda 2030 y los Objetivos de Desarrollo Sostenible (ODS) que, desde el 1 de enero de 2016, rigen los esfuerzos de los países para lograr un mundo sostenible en el horizonte de 2030. Los ODS abarcan 17 objetivos y 169 metas, así como una declaración de intenciones que articula los principios de integración, universalidad, transformación y una asociación global en pos del desarrollo humano dentro de los límites biofísicos de la Tierra.

Su implementación no está parametrizada puesto que, aunque se trata de un empeño colectivo y global, corresponde a cada país decidir cómo aplicarlo y priorizar sus objetivos y metas dentro de un marco común. Esta realidad hace que la agenda de cada país, conocida como Agenda 2030, pueda ser divergente en las formas, pero no en el fondo.

En definitiva, para numerosos agentes económicos la sostenibilidad, como algo general, y los ODS como algo más tangible, proporcionan ya una hoja de ruta hacia un desarrollo más duradero y un mercado de oportunidades en crecimiento, a la vez que demuestran que mejorar la sostenibilidad no necesariamente tiene un coste para ellos, sino que puede proporcionar beneficios significativos a largo plazo que son el foco de las finanzas sostenibles.

Pocas semanas después de la Cumbre de Naciones Unidas sobre Desarrollo Sostenible, tenía lugar en París la histórica Conferencia de las Partes (COP-21), en la que se alcanzó el conocido como Acuerdo de París. El texto, acordado por 195 países además de la Unión Europea, sustituyó al Protocolo de Kioto (1997) que en su momento constituyó el primer acuerdo global para reducir las emisiones de gases de efecto invernadero.

\footnotetext{
${ }^{1}$ https://www.un.org/sustainabledevelopment/es/development-agenda/

2 https://unfccc.int/process-and-meetings/the-paris-agreement/the-paris-agreement
} 
Dichas emisiones hace tiempo que han sido identificadas por la comunidad científica como uno de los principales causantes del incremento tendencial de la temperatura en la tierra. Sobre la base de un diagnóstico de las amenazas asociadas al calentamiento global que dejaba poco lugar a la complacencia ${ }^{3}$, se establecieron unos objetivos de reducción de emisiones de gases de efecto invernadero y de adecuación de los flujos de financiación de las economías a esos propósitos. Desde entonces, las evidencias han dejado corto aquel diagnóstico, verificándose la persistencia de niveles elevados de emisiones, el correspondiente ascenso de las temperaturas, así como catástrofes naturales de diversa índole con el denominador común de su atribución a ese creciente deterioro medioambiental. Un análisis precursor, desde una perspectiva económica fue el trabajo del profesor de la London School of Economics, Nicholas Stern, difundido en octubre de 2006. En él se advertía, como se comenta en Ontiveros (2019), sobre la magnitud de los costes derivados del cambio climático, de las amenazas desde una perspectiva fundamentalmente económica, incorporando nuevas formas de análisis del impacto y nuevos métodos, que concluían en la reducción del crecimiento económico global.

\section{LA AGENDA EN LAS EMPRESAS PIONERAS}

\subsection{FINANZAS SOSTENIBLES Y EMPRESAS RESPONSABLES: EL PARADIGMA BLACKROCK}

Es alentador ver que muchos de los primeros agentes que tomaron conciencia y desarrollaron acciones en el ámbito de las finanzas y la sostenibilidad eran del sector privado. Aun así, será necesario activar muchas más acciones y de mayor calado que las actuales, y que éstas se materialicen de forma efectiva en modelos de negocio generalistas dado que, de no materializarse de forma autorregulada, puede ser necesario que se tenga que establecer nueva normativa para que aceleren su transformación.

Las razones de ese movimiento temprano de algunos agentes financieros cabe atribuirlas, en parte a un cierto efecto anticipador de esas exigencias regulatorias y/o informativas que tarde o temprano iban a llegar, pero sobre todo a una evaluación, por parte de los agentes financieros más dinámicos, de los riesgos y oportunidades que iban a emanar del ineludible cambio hacia un modelo de crecimiento sostenible.

Cada vez son más las entidades, sobre todo en el ámbito de la gestión de activos, que adoptan estrategias mucho más proactivas de medición y comunicación en relación con el cambio climático y la huella de carbono, llegando en algunos casos a comprometerse con objetivos cuantificables de reducción de su exposición a activos causantes de dicha huella.

Esas políticas de diferenciación en la transparencia, o incluso en el compromiso de reducción de activos expuestos, pueden ser valiosas también en una perspectiva de optimización del binomio rentabilidad/ riesgo, como se pone de manifiesto en algunos estudios recientes, y de forma especial en un reciente informe de BlackRock (2018). En el mismo se analiza "la performance" de varias carteras de acciones,

\footnotetext{
${ }^{3}$ La comunidad científica advierte que si la cantidad de dióxido de carbono que hay en la atmósfera supera las 450 partículas por millón (ppm), lo que equivale a asumir un incremento máximo de en torno a $2^{\circ} \mathrm{C}$ de la temperatura del planeta sobre valores pre-industriales, los efectos desastrosos del cambio climático podrían descontrolarse
} 
clasificadas en función de la intensidad en carbón de su actividad con respecto a las ventas anuales. Como puede comprobarse en el gráfico 3, esos resultados son tanto mejores cuanto mayor ha sido el avance de las compañías en reducir la intensidad de carbón en las carteras.

Gráfico 3. Rentabilidad de la renta variable por intensidad de carbono, 2012-2018

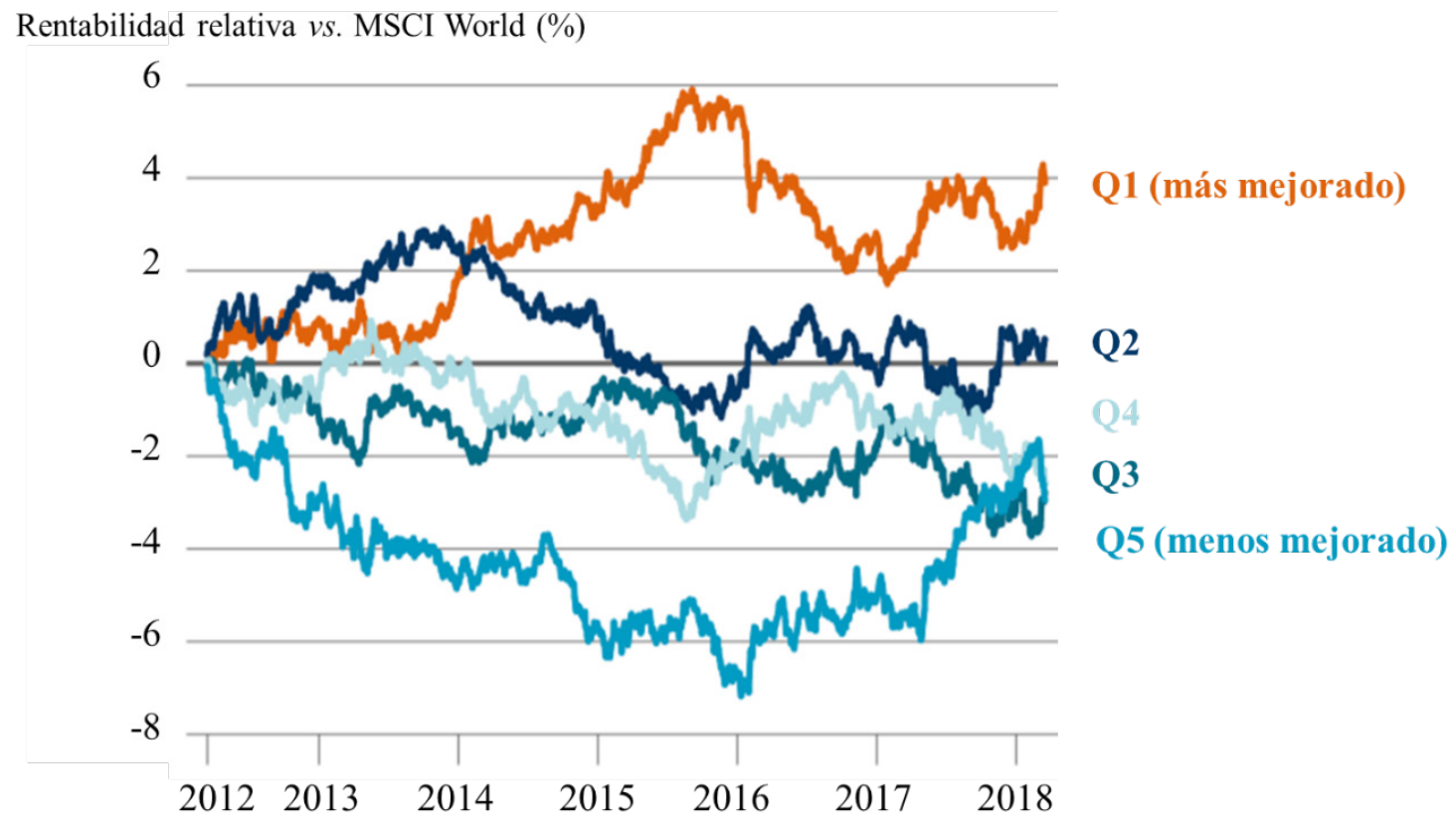

Fuente. Blackrock. 2018

Quizás por esa explícita compatibilidad entre rentabilidad y comportamiento sostenible es por lo que el propio Blackrock, el inversor institucional con mayor volumen de activos bajo gestión del mundo, viene advirtiendo de forma recurrente a los principales responsables de las empresas de las que posee acciones sobre la necesidad de más transparencia e información sobre los efectos del cambio climático en sus actividades, en sus estados financieros, en definitiva.

En la misma dirección, cabe destacar la declaración que un conjunto de 60 inversores institucionales (responsables de activos bajo gestión por 10 billones de dólares) realizaron en 2018, a través del Financial Times, dirigida a "sus" empresas vinculadas al negocio del gas y petróleo. Les reclaman explicaciones sobre cómo piensan atender la necesaria transición energética hacia una economía sin emisiones netas de carbono que se acordó en París de 2015 por más de 200 estados y sobre la que varios países han empezado ya a legislar. El sector de combustibles fósiles no es muy dependiente del mercado de bonos para su financiación, por lo que la exigencia de los mercados financieros hacia una transición de su actividad viene fundamentalmente de los mercados de acciones, de ahí la importancia del posicionamiento de estos inversores. 


\subsection{EL SISTEMA FINANCIERO, CLAVE PARA LA SOSTENIBILIDAD}

Ese especial protagonismo del sector financiero ante la sostenibilidad, y especialmente la lucha contra el cambio climático, emerge de su potencial para anticipar efectos futuros y establecer precios que puedan actuar como señales para guiar tanto las políticas públicas como las decisiones a tomar por los agentes privados. Al fin y al cabo, la esencia de los mercados financieros es su capacidad de valoración relativa entre el presente y el futuro, y eso es precisamente lo que se necesita para superar la "tragedia del horizonte", tal como la definió en 2015 Mark Carney, a la sazón presidente del Financial Stability Board, y del Banco de Inglaterra, quien primero llamó la atención sobre las implicaciones del cambio climático para la estabilidad financiera. Subrayaba cómo los evidentes daños del cambio climático no generaban incentivos para que los actuales responsables adoptaran decisiones para su neutralización, dado que sus principales daños los sufrirán las próximas generaciones. Esa premonición de Carney se quedó corta: el horizonte ya está aquí; los daños ya los están sufriendo las generaciones presentes.

Por otra parte, ese claro alineamiento del sector financiero con la transición hacia una economía libre de emisiones se ve apoyado por las abundantes evidencias disponibles respecto a la positiva valoración que los mercados asignan a empresas comprometidas con dicha transición, así como respecto a las menores tasas de morosidad que las entidades financieras pueden esperar de los créditos concedidos para financiar actividades "verdes".

En este segundo ámbito, el estudio más reciente, y sin duda el más comprensivo de los llevados a cabo hasta la fecha, es el realizado por el Banco de Inglaterra (2020) a principios del presente año, y analizando microdatos para una muy amplia muestra (más de un millón) de créditos hipotecarios vivos en dicho país. Con técnicas estadísticas suficientemente robustas, el estudio concluye que las hipotecas concedidas para viviendas que satisfacen los requerimientos de eficiencia energética incorporan una probabilidad de impago en torno a un $20 \%$ inferior que las correspondiente a viviendas que no satisfacen esas exigencias. Sobre la base de esos resultados se concluye con una recomendación importante: la eficiencia energética ha de ser considerada un factor a la hora de estimar la probabilidad de impago (la conocida como PD), y sea tenida en cuenta por las entidades prestamistas en la fijación del tipo de interés de concesión.

Al hilo de dichas evidencias y recomendaciones, no es casual que sea también el Banco de Inglaterra uno de los primeros que ha tomado ya la iniciativa de someter a las entidades financieras a un primer ejercicio de resistencia (Stress Test) frente a los riesgos asociados al cambio climático, iniciativa que sin duda será seguida por la mayoría de supervisores bancarios, desde esa constatación enunciada por Mark Carney, de que el cambio climático afecta directamente a la estabilidad financiera, y como tal debe ser objeto de medición y vigilancia por parte de los supervisores bancarios.

Pero no solo el sistema bancario, sino los mercados financieros en general, han mostrado un claro alineamiento con esa transición hacia una economía verde. De hecho, un estudio conjunto del BCE y el Banco Europeo de Reconstrucción y Desarrollo, BCE_BERD (2019) pone de manifiesto que dicha transición se ve más favorecida por un sistema financiero basado en un mayor peso de los mercados de capitales que de la intermediación bancaria tradicional. De dichos resultados extraen la recomendación de apoyar el desarrollo de mercados de valores, mucho más proclives a financiar actividades de más riesgo, en general más intensivas en innovación, así como las relacionadas con la transición hacia una economía verde y más circular. 
Son numerosos los estudios sobre la relación entre criterios ASG (ambientales, sociales y de gobierno corporativo) y rentabilidad financiera, pero por su carácter de metaanálisis cabe señalar el elaborado por Friede et al. (2015). En el mismo se señala que aproximadamente el $90 \%$ de los estudios encuentran una relación ASG y desempeño financiero corporativo no negativa. Más importante aún, la gran mayoría informa sobre resultados positivos.

Ese favorable comportamiento de los activos financieros "verdes", tanto en acciones como en bonos, se ha demostrado especialmente efectivo en el marco de la crisis desencadenada por la COVID-19, que ha provocado intensas caídas en la mayoría de los mercados de valores. Pues bien, esas caídas han sido significativamente menores en el caso de los valores más alineados con la sostenibilidad y el cambio climático.

Comenzando por el mercado de acciones, ha sido suficientemente documentada la existencia de lo que se denomina un "Greenium negativo", es decir una prima por riesgo negativa asociada a los valores con un mayor componente verde (de ahí el acrónimo "greenium", o "green premium") en su actividad empresarial. Esa prima de riesgo negativa se traduce en una sistemática mejor valoración de las compañías con un mayor componente verde, de tal manera que, según la Comisión Europea (2019), en el horizonte de la última década una cartera de acciones de empresas "verdes" habría generado un $8 \%$ acumulado de rentabilidad diferencial.

Pero, adicionalmente, la inversión en "acciones verdes" se ha mostrado más resistente frente a las importantes caídas de valor de las acciones en el marco de la crisis provocada por la emergencia sanitaria de la COVID-19. En ese contexto cabe destacar el favorable comportamiento diferencial de aquellas compañías con un perfil de sostenibilidad robusto desde el punto de vista ambiental, social y de gobierno corporativo (ASG). Para ello, hemos analizado tres familias de índices sostenibles, con diferentes grados de exigencia en cuanto a sostenibilidad, realizando el estudio a nivel global y regional para evitar posibles sesgos geográficos, Pues bien, tanto en un ámbito global, como en Estados Unidos, Europa, o los países emergentes, los índices de acciones con un mayor componente verde en sus integrantes, han registrado un descenso de precios sustancialmente inferior al de los agregados de mercado.

En lo que a riesgo se refiere, la inversión sostenible medida a través de volatilidad no presenta diferencias significativas, incluso en algunos casos, los índices con características ASG presentan mayores niveles de volatilidad como consecuencia del menor número de valores en cartera (los índices MSCI SRI agrupan el $25 \%$ de activos del índice tradicional), y por tanto, un menor efecto diversificación.

Sin embargo, cuando utilizamos otras medidas como el drawdown o máxima caída, vemos cómo los índices sostenibles vuelven a presentar mejores resultados. En todos los casos, el máximo drawdown registrado en los últimos cinco años se da en marzo de 2021, con la aparición de la pandemia, y de nuevo, tanto a nivel global como regional, los índices sostenibles presentan caídas menos pronunciadas que sus índices tradicionales. Y es que precisamente estos eventos de cola, que puedan generar pérdidas extraordinarias en una compañía, son los que trata de mitigar la inversión decidida con criterios de sostenibilidad.

Esa referencia a eventos de cola nos lleva necesariamente a hacer una última reflexión sobre el sector de seguros, sin duda el más vinculado a eventos de cola. Como ilustrábamos en la sección 2 , el incremento de eventos extremos asociados al cambio climático se ha traducido en un incremento caso exponencial de las pérdidas provocadas por los mismos, de las que apenas un tercio se encontraban aseguradas. Ello abre una necesidad (a la vez reto y oportunidad de negocio) para que el sector asegurador asuma una posición de claro liderazgo en la transición hacia un modelo productivo más sostenible. 


\section{EL MERCADO DE BONOS VERDES}

\subsection{DESARROLLO EXPONENCIAL DEL MERCADO: PROFUNDIDAD Y DIVERSIDAD DE EMISORES}

Uno de los elementos más representativos del creciente desarrollo de las finanzas sostenibles es la extensión registrada por los denominados Bonos Verdes. Surgidos en 2007 gracias a una emisión del Banco Europeo de Inversiones, su actividad fue evolucionando en sus primeros años de vida al amparo fundamentalmente del impulso por los organismos multilaterales. Desde 2013, con las primeras emisiones de empresas (la francesa EDF) y entes públicos (Estado de Massachusetts), se abre claramente el espectro de emisores, en el que irán ganando peso emisores privados de diferentes países, siendo España uno de los más activos en dicho segmento, tanto con emisiones públicas, como sobre todo privadas, destacando Iberdrola, uno de los más activos emisores privados en el mundo.

Gráfico 4. Bonos verdes: emisiones anuales y saldo acumulado

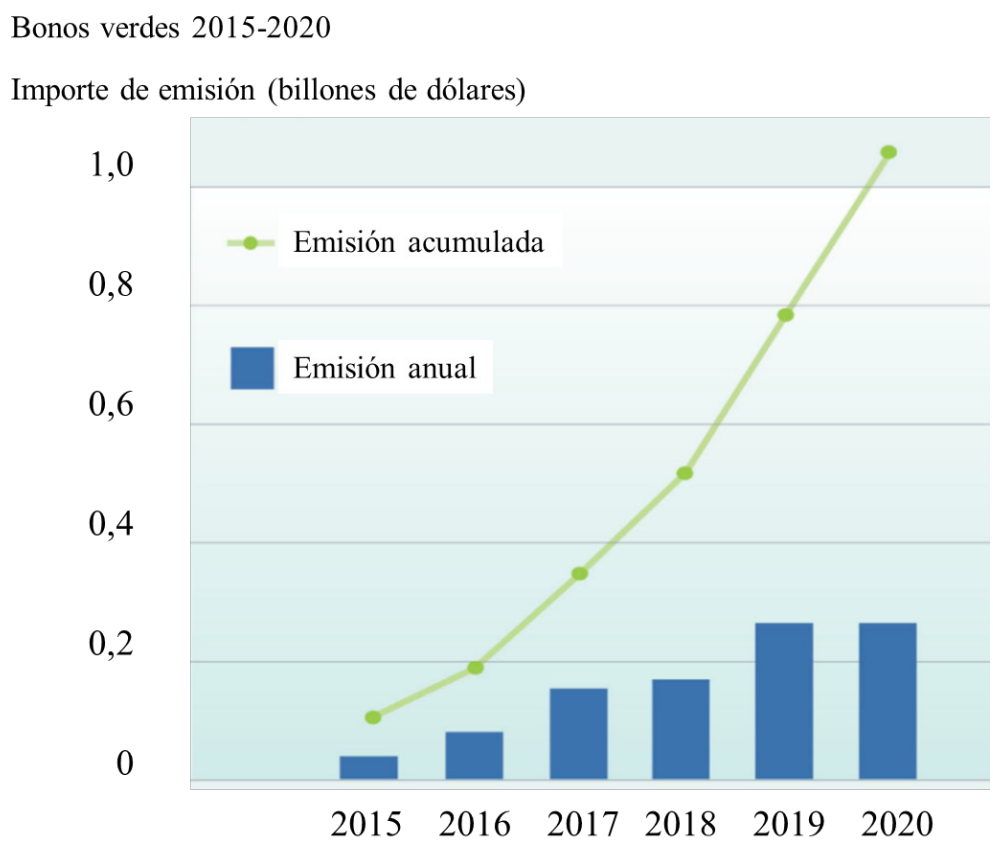

Fuente: Climate Bonds Initiative (2021)

Autorregulado en base a los acuerdos adoptados en el marco de los denominados Green Bond Principles $(G B P)^{4}$, puede ser considerado como un elemento clave en la creciente aceptación de las finanzas sostenibles, tanto por su impacto positivo sobre aspectos medioambientales como por su capacidad demostrada de atracción de capital privado para financiar necesidades globales. Además, su evolución a lo largo del tiempo ha permitido el desarrollo de otro tipo de productos financieros similares (bonos sociales, préstamos verdes, préstamos sociales) que acercan cada vez más la sostenibilidad al sistema financiero desde el punto de vista del diseño de producto y el impacto del mismo.

\footnotetext{
${ }^{4}$ https://www.icmagroup.org/green-social-and-sustainability-bonds/green-bond-principles-gbp/
} 
Porque un elemento clave de las finanzas sostenibles es su creciente innovación y diversificación de oportunidades. Por ejemplo, los bonos y préstamos ligados a sostenibilidad vinculan el tipo de interés que aplican a la consecución de algún objetivo particular o general de sostenibilidad de la entidad receptora de la financiación. Se ha estimado que en el año 2019 , este tipo de operaciones supuso más de un $10 \%$ del total de financiación sostenible emitida, cuyo volumen acumulado superó los 800.000 millones de dólares (ver gráfico 4).

\subsection{COMPORTAMIENTO DIFERENCIAL DE LOS BONOS VERDES}

En paralelo a ese crecimiento de la actividad emisora en instrumentos financieros sostenibles, cobra una gran relevancia la medición del comportamiento de dichas inversiones en términos del binomio entre rentabilidad y riesgo. Las evidencias existentes al respecto son claramente favorables: los bonos verdes han generado, de forma consistente durante la década transcurrida desde la crisis financiera, una rentabilidad acumulada que supera, en casi 8 puntos porcentuales, a la registrada por bonos equivalentes sin dicha consideración.

A ese mejor comportamiento como inversión de los bonos verdes de forma estructural, cabe añadir su mejor comportamiento en momentos de severa crisis, como la generada por la emergencia sanitaria. En dicho marco, los bonos verdes han puesto de manifiesto una capacidad de resistencia mucho mayor que el resto de bonos. Concretamente, en el contexto de caídas generalizadas que experimentaban los bonos corporativos al desatarse la pandemia, la caída de precio en los bonos verdes fue menos de la mitad que en el agregado de bonos de alta calidad crediticia.

Sobre la base de esas evidencias referidas al mejor comportamiento relativo de los bonos verdes, tanto en un contexto de normalidad y recuperación económica -una década completa desde la anterior crisis-, como en el marco de la crisis provocada por la emergencia sanitaria (COVID-19), ya no cabe albergar dudas sobre el valor de dicho tipo de instrumentos en cualquier cartera de inversión, como así han asumido ya una buena parte de los principales gestores de activos, que asumen crecientes compromisos en cuanto al peso de los bonos verdes en sus carteras.

En esta misma dirección, cabe destacar que el Banco Central Europeo, en sus nuevas políticas de inversión en bonos, en el marco de las medidas para la superación de la crisis de la COVID-19, asume de forma proactiva esa presencia de bonos verdes en sus decisiones de compra de activos, y de forma especial en las compras asociadas al denominado PEPP (Pandemic Emergency Purchase Program).

Un compromiso en esa dirección constituye el mejor reconocimiento de que la lucha contra la pandemia y contra el cambio climático son objetivos, no solo compatibles, sino con importantes complementariedades desde la perspectiva de los mercados financieros. De ese claro compromiso del BCE con la sostenibilidad y el cambio climático da buena fe la intensa política de comunicación y de coordinación de actividades en el seno del BCE, como su presidenta ha dejado claro en recientes intervenciones, así como en la creación de un "Climate Change Center" en el seno de dicha institución'.

${ }^{5}$ Véase Lagarde (2021) 


\section{EUROPA A LA VANGUARDIA}

\subsection{PROTAGONISMO FRENTE AL RESTO DE LAS POTENCIAS}

Cuando se han cumplido cinco años de la suscripción del Acuerdo de París, cabe concluir con una cierta decepción, en la persistencia de niveles elevados de emisiones, de ascenso de las temperaturas, así como catástrofes naturales de diversa naturaleza con el denominador común de su atribución a ese creciente deterioro medioambiental.

La decepción derivada del desigual cumplimiento de aquellos compromisos está siendo compensada, sin embargo, por algunos elementos esperanzadores. El más importante es la extensión de la conciencia de la emergencia climática entre ciudadanos y empresas en todo el mundo, como ya hemos comentado en la sección 3.

En el ámbito de las administraciones públicas, la decepción que ha supuesto la posición estadounidense en los cuatro años de mandato de Trump -y que la nueva Administración Biden ya se ha apresurado a corregirha sido compensada por la entusiasta posición china, y muy especialmente europea, verdadera avanzadilla en la lucha contra el cambio climático.

Con el objetivo de "convertir a Europa en el centro de gravedad de la inversión global en una economía baja en carbono, eficiente en la utilización de los recursos y circular" la Comisión Europea presentó en marzo de 2018 su Plan de Acción para la financiación del crecimiento sostenible, o Plan de Acción en finanzas sostenibles (Comisión Europea, 2018), en gran medida siguiendo las recomendaciones del grupo de expertos analizadas en la sección anterior.

El plan de acción incluye 10 iniciativas, entre las que cabe destacar:

- Desarrollar una Taxonomía específica sobre "sostenibilidad" a nivel europeo que se integre progresivamente en la legislación de la UE para proporcionar una mayor seguridad jurídica a inversores y otros agentes, aunque bajo un criterio de neutralidad tecnológica.

- Crear estándares y etiquetas vinculadas a productos financieros verdes. Con ello se confía en que el creciente interés de los consumidores por este tipo de etiquetas se traslade a los productos financieros (como fondos de inversión especializados en economía baja en carbono, bonos verdes o incluso hipotecas verdes que se desarrollen bajo su amparo), dándoles una visibilidad, mecanismos de control y gobernanza que debería favorecer una mayor demanda de los mismos.

- Incorporar la sostenibilidad en los requerimientos prudenciales. La Comisión va a desarrollar un análisis sobre los requisitos de capital más adecuados que deben tener los "activos sostenibles" en poder de bancos y compañías de seguros.

Caben pocas dudas, por tanto, de la voluntad de las instituciones europeas de alinear la tensión transformadora de las finanzas con la senda de la sostenibilidad de forma irreversible. De compatibilizar, en definitiva, la sostenibilidad con la modernización financiera, que incluiría también un mayor equilibrio entre financiación directa y financiación intermediada, como se analiza en Berges-Ontiveros (2018). 


\subsection{DEL PACTO VERDE (GREEN NEW DEAL) A LA RECUPERACIÓN VERDE POST-COVID}

La Unión Europea ya daba claras muestras de su liderazgo mundial en sostenibilidad con su iniciativa del Pacto Verde (Green New Deal) en diciembre de 2019, que suponía una clara intensificación y aceleración de los compromisos asumidos en el Acuerdo de París. Concretamente, se aceleraba hasta un 55\% (con respecto a los niveles de 1990) el compromiso de reducción de emisiones al horizonte de 2030, y se asume un objetivo de cero emisiones netas al horizonte de 2050.

Este Green New Deal pretende transformar la economía europea en plenamente sostenible, cambiando su modelo social y económico y proporcionando, al mismo tiempo, los recursos económicos que permitan una transición justa. Para ello, la Comisión Europea propuso crear un fondo de transición justa para las regiones más dependientes de los combustibles fósiles de hasta 100.000 millones de euros, aunque algunas estimaciones apuntan a que dicho importe era apenas un tercio de lo que realmente se necesita.

Ha tenido que ser la pandemia, y en particular la contundente respuesta de la Comisión Europea para superar los estragos causados por la misma en las economías europeas, la "excusa" para una movilización de recursos mucho más acorde con esos ambiciosos objetivos del Green New Deal. En particular, al menos una tercera parte de los 750.000 millones de euros con que está dotado el "Next Generation EU" (NGEU) se asignarán a proyectos que garanticen la satisfacción de ese propósito.

EI NGEU constituye un presupuesto europeo excepcional y temporal, que pretende captar en los mercados hasta 750.000 millones de euros con la emisión de deuda conjunta emitida por la Comisión Europea en nombre de la UE para destinarlo a un programa de inversión y reformas que combine dos objetivos:

- La recuperación económica tras la pandemia. EI PIB va a sufrir su mayor descenso en tiempos de paz (con notables divergencias por países), mientras el paro en Europa volverá a acercarse al 10\% de la población activa. El impulso a la inversión que facilitará el NGEU acentuará el rebote que experimentará la economía en 2021.

- La transformación de la economía europea. La filosofía de la acción que ha adoptado la UE busca, en la mejor tradición de la política económica, aunar la satisfacción de lo urgente con la atención al medio y largo plazo. Así, las directrices del NGEU apuntan a la necesidad de reformar y cambiar aspectos y sectores básicos del funcionamiento de las economías para i) reforzar las áreas cuya debilidad ha quedado patente, ii) acelerar la transición energética y la lucha contra el cambio climático y iii) desplegar las potencialidades de las tecnologías digitales.

Siguiendo con estas directrices, el Gobierno español presentó el 7 de octubre el Plan de Recuperación, Transformación y Resiliencia, que pretende concentrar el esfuerzo de reforma e inversión en el trienio 20212023 llevando la inversión pública hasta el 6\% en media y movilizando también inversión privada. El Plan se articula en torno a cuatro ejes transversales (transición ecológica, transformación digital, cohesión social y territorial e igualdad de género) y diez políticas palanca de reforma estructural, con dotaciones estimadas en términos de porcentajes de recursos respecto al total. Según las estimaciones realizadas por la Comisión Europea, España podría recibir hasta 140.000 millones de euros, repartidos casi al $50 \%$ entre transferencias y préstamos, lo que equivale al 11\% del PIB de 2019. 
España está en disposición de recuperar el tiempo perdido. La OCDE, en su último informe de perspectivas destacaba en el capítulo español, el compromiso de las autoridades para intensificar las inversiones en energías renovables, en eficiencia energética y en el transporte, anticipando la contribución de esas acciones a la recuperación económica y del empleo. La esperada aprobación y entrada en vigor de la Ley de Cambio Climático y Transición Energética (PLCCyTE) debe consolidar esas aspiraciones no solo de recuperación verde, sino de contribución a la transformación y modernización de nuestra economía. A la satisfacción de esas pretensiones deberán contribuir no solo la instalación de nueva capacidad de origen renovable, sino la generación de proyectos transversales en distintos sectores de la economía, desde los vinculados a la movilidad al sector turístico, pasando por la eficiencia energética en todo tipo de edificaciones.

No son pretensiones inalcanzables ni faltan fundamentos en las que asentarlas. En nuestro país se encuentran operadores empresariales y financieros que han asumido hace tiempo esa necesidad. Que anticiparon inteligentemente esa exigencia global y hoy mantienen posiciones de liderazgo mundial en la generación de energías renovables y en el empleo de modalidades de financiación verde y socialmente sostenibles. La estrecha colaboración público-privada que ha de presidir la concreción de proyectos de inversión en esos ámbitos puede ser determinante para que la inversión verde genere los efectos multiplicadores pretendidos. La asignación de recursos a la inversión en infraestructuras para la electrificación, en energías renovables, en eficiencia energética y en investigación en tecnologías limpias pude impulsar tanto más el crecimiento económico y del empleo cuanto mayor sea esa coordinación entre agentes. Así lo ponen de manifiesto diversos estudios, como el elaborado entre la Agencia Internacional de la Energía (AIE) y el FMI. Trabajos de la AIE destacan, además, que la persistencia del empleo creado por la inversión verde es superior al promedio.

Si en nuestro país se asume con el suficiente respaldo político esa prioridad de modernización económica desde la base de la transición energética, de todo punto compatible con esa otra prioridad de extensión de la digitalización, no pocos sectores hoy diezmados por la reclusión obligada por la pandemia podrán hacer de la necesidad virtud. También podrán reforzarse los atractivos de nuestro país como un destino sano en su más amplia acepción, no solo para los visitantes turísticos de calidad, cada día más exigentes con las condiciones medioambientales, sino también para la captación de flujos de inversión extranjera directa e incluso talento externo, en torno a esos proyectos de inversión verde. 


\section{REFERENCIAS BIBLIOGRÁFICAS}

- Alessi, Lucia; Ossola, Elisa; y Panzica, Roberto (2019). The Greenium matters: greenhouse gas emissions, environmental disclosures, and stock prices, Working Papers 2019-12, Joint Research Centre, European Commission.

- Berges, Ángel; Ontiveros, Emilio (2018). De la Unión Bancaria a la Unión de Mercados de Capitales, en Información Comercial Española, n 902, Mayo 2018, págs. 95-107

- BlackRock Investment Institute (2018) Sustainable investing: a "why not" moment. Global Insights. Mayo

- Bolton, Patrick; Despres, Morgan; Pereira da Silva, Luiz Awazu; Samama, Frédéric; y Svartzman, Romain (2020). The green swan. Central banking and financial stability in the age of climate change. BIS. Banque de France. Enero.

- Climate Bonds Initiative (2021) Record \$269.5bn green issuance for 2020: Late surge sees pandemic year pip 2019 total by $\$ 3 b n$

- De Haas, Ralph y Popov, Alexander (2019) Financial Development and Industrial Pollution EBRD Working Paper 217. European Central Bank

- Fondo Monetario Internacional (2020), World Economic Outlook. Octubre

- Friede et al. (2015) ESG and financial performance: aggregated evidence from more than 2000 empirical studies. Journal of Sustainable Finance and Investment, 5:4, págs. 210-233

- Guin, Benjamin y Korhonen, Perttu. (2020) Does energy efficiency predict mortgage performance? Bank of England. Staff Working Paper No. 852. January.

- Lagarde, Chistine (2021): "Climate change and Central Banking", Keynote speech by Christine Lagarde, President of the ECB, at the ILF conference on Green Banking and Green Central Banking

- Ontiveros, Emilio (2019), Excesos. Amenazas a la prosperidad global. Editorial Planeta.

- Serhan Cevik y João Tovar Jalles. Feeling the Heat: Climate Shocks and Credit Ratings Fondo Monetario Internacional. Working Paper 2020/286. Diciembre. 
